\title{
Refining the Predictive Potential of the Early Repolarization Pattern in Myocardial Infarction: An Elusive Endeavour
}

\author{
Moisés Rodríguez-Mañero ${ }^{\mathrm{a}-\mathrm{c}}$ José Ramón González-Juanatey ${ }^{\mathrm{a}-\mathrm{c}}$ \\ ${ }^{a}$ Cardiology Department, Complejo Hospital Universitario de Santiago, Santiago de Compostela, Spain; \\ b Instituto de Investigación Sanitaria (IDIS), Universidad de Santiago de Compostela, Santiago de \\ Compostela, Spain; ${ }^{C}$ Centro de Investigación Biomédica en Red de Enfermedades Cardiovasculares \\ (CIBERCV CB16/11/00226 - CB16/11/00420), Madrid, Spain
}

Early repolarization (ER) has been a matter of heated debate since its first description. Theories regarding its relevance range from an innocuous finding in healthy young subjects to its association with life-threatening ventricular tachyarrhythmias (VTA/ventricular fibrillation $[\mathrm{VF}]$ ), both in patients with and without structural heart disease (including patients with coronary artery disease) [1-5]. A proposed mechanism for the ER pattern is an imbalance in the ion channel system, resulting in variable refractoriness of multiple myocardial regions and varying excitability in the myocardium $[2,3]$. This can produce a voltage gradient between myocardial regions, which is believed to cause the major hallmarks of the ER pattern; i.e., ST-segment elevation and QRS notching or slurring.

Although for the most part, the ER pattern is globally accepted as a marker of risk, several doubts and methodological concerns have surrounded the supporting data. Importantly, the exact definition of ER has varied according to different investigators. In the seminal study by Haïssaguerre et al. [3], ER was defined as an elevation of the QRS-ST-segment junction (J-point) in at least 2 leads, within the same territory, with the amplitude of the Jpoint elevation equal to at least $0.1 \mathrm{mV}$ above the baseline level, as either QRS slurring or notching. Importantly, the

\section{KARGER}

(c) 2019 S. Karger AG, Basel

E-Mail karger@karger.com

www.karger.com/crd amplitude and slope of the ST-segment were not part of the definition. In subsequent studies, Tikkanen et al. [4, 5] incorporated the degree of so-called J-point elevation, which was stratified at levels of 0.1 and $0.2 \mathrm{mV}$. Moreover, they took into account the ST-segment slope, showing that a horizontal or downward sloping ST-segment was associated with a greater arrhythmic risk [5]. Thus, there has been considerable variation in the definition of ER, as well as some controversy regarding the term itself. This has led to some divisive results and nonuniform methodology. Despite this crucial methodological limitation, the phenomenon remains a matter of interest as a promising risk factor for predicting arrhythmic risk, at least in the setting of patients with structural heart disease (in addition to the degree of left ventricular dysfunction and Killip class).

In the pursuit of more precise patient categorization, the present meta-analysis by $\mathrm{Xu}$ et al. [6] attempts to investigate the relationship between the ER pattern - and importantly, its detailed characteristics - and the incidence of VTAs in patients with acute myocardial infarction (AMI). Ten studies (out of an initial search of 476 potentially relevant studies) with a cumulative total of 2,672 participants were included in the final analysis. All studies but one involved a sample size that was $\geq 100$. The 
mean age of the participants in the ten studies ranged from 48.4 to 69 years. Four studies only recruited patients after onset of ST elevation myocardial infarction, and seven studies included patients who underwent percutaneous coronary intervention.

The analysis raises several important conclusions. First of all, the meta-analysis showed that ER on electrocardiogram correctly predicts VTAs in patients after onset of AMI, irrespective of the type of AMI. Notably, although the amplitudes of J-point elevation were divergent and ranged from 0.5 to $2.0 \mathrm{mv}$, J-point elevation did not potentiate the risk of VTAs in patients with AMI. With regard to the J-point elevation, there is considerable variation in the use of the term J-point. For many cardiologists, this is taken as the onset of the ST-segment [7], which may equate with the termination of an end-QRS notch, whereas others use the term for the peak $[1,2]$ or the onset of an end-QRS notch [8]. The majority of publications at the present time [1-3] adopt the amplitude of J peak (Jp). This has led to the elaboration of a consensus document that suggests that the following terminology should be used [8]: (1) J onset (Jo) should denote the onset of a notch; (2) (Jp) should denote the peak of a notch or onset of a slur; and (3) J termination (Jt) should denote the end of a notch or slur. This precise new terminology is not analyzed in the papers constituting the meta-analysis due to lack of specification in the included studies. Consequently, the conclusions of the analysis will have to be reconfirmed and adapted to the new terminology in successive studies.

Authors of included papers also analyzed the role of ER in the lateral lead location. These additional findings are important in guiding clinicians on the precise electrocardiogram leads that should be incorporated into future prognostic algorithms. This stems from the pathophysiological mechanism underlying the arrhythmogenic role of the ER pattern. As aforementioned, the EP pattern reflects a distinct action potential notch mediated by transient outward potassium current (mediated by the Ito, IK-ATP, and IK-Ach channels) that produces transmural electrical heterogeneity between the endocardium and the epicardium during early ventricular repolarization. The repolarization dispersion enlarged by this heterogeneity could lead to phase 2 re-entry capable of initiating VF [9-12]. Remarkably, the present study reports an increased risk in patients with inferior ER but not for those with lateral ER (although as stated in the study, the individual leads that constituted lateral leads were slightly different). As the authors postulated, this finding may result from the greater predominance of Ito current in the right ventricular epicardium $[6,13]$. Conversely, in the analysis

Early Repolarization Pattern in

Myocardial Infarction by $\mathrm{Xu}$ at al. [6], ER predicted VTAs irrespective of the location of AMI pointing towards a global phenomenon more than location-specific ramifications. Theoretically, patients who had a myocardial infarction have scar tissue in the myocardium that could become a substrate for ventricular tachycardia [13]. A reasonable assumption is that phase 2 re-entry and scar tissue play an important role in the development of sustained VT/VF as the trigger and substrate, respectively, resulting in the higher prevalence of VT/VF in the setting of AMI in patients with ER than in those without.

The study by $\mathrm{Xu}$ et al. [6] raises another important conclusion. It is conceivable to reason that the ER pattern could be affected by an ongoing AMI, which adulterates its predictive role. However, the prognostic value of ER presence in predicting VTA was even higher before the occurrence of AMI (OR 5.58, 95\% CI 3.41-9.12) than after (OR 3.02, 95\% CI 2.19-4.15). Moreover, authors report significantly higher predictive values in the short follow-up period (defined as $<30$ days) as compared with the long follow-up duration ( $\geq 30$ days). ER may sequentially appear and disappear during clinical follow-up [14]. As such, further investigations should describe the precise timing of pattern appearance. Accordingly, it might be that discrepancies observed in previous studies could be due to different detection windows.

There are several important limitations that need to be outlined. As the author pointed out, the use of $\beta$-blockers and statins was relatively low for the cohort given existing guidelines for post-AMI management. Moreover, the rate of percutaneous coronary intervention was unacceptably low in two of the studies (46.5\% [15] and 51.6\% [16]) and also, anti-arrhythmic drug use was not taken into account at the time of the analyses. These facts undoubtedly might have affected the incidence of VT/VF or sudden death occurrence and could be vastly different at the present time of early reperfusion strategy and optimal medical treatment. Moreover, the conclusions of the present study need to be analyzed by the limitations intrinsic to metaanalysis: the poor quality of some included studies (although 9 out of the 10 studies included in the study by Xu et al. [6] were considered high quality, and only one study was considered moderate quality), the heterogeneity between studies included, and the failure to address publication bias. Importantly meta-analyses are limited by the chosen subgroup analysis within their included studies. For instance, in the present analysis, only 3 out of 10 studies analyzed employed sustained VF as an endpoint event, whereas other studies utilized a more generic endpoint defined as "VTAs." 
Despite these limitations, the authors should be commended for undertaking such an exhaustive and comprehensive analysis. The current study adds useful information to our collective knowledge base and reinforces the findings of previous analyses of ER. We agree with the authors that more studies, preferably prospective ones, are now required to provide definitive evidence relating to the prognostic significance of ER in patients with AMI. For the time being, the present study supposes a step forward.

\section{Disclosure Statement}

The authors have no conflicts of interest to disclose.

\section{References}

1 Rosso R, Glikson E, Belhassen B, Katz A, Halkin A, Steinvil A, et al. Distinguishing "benign" from "malignant early repolarization": the value of the ST-segment morphology. Heart Rhythm. 2012 Feb;9(2):225-9.

2 Rosso R, Kogan E, Belhassen B, Rozovski U, Scheinman MM, Zeltser D, et al. J-point elevation in survivors of primary ventricular fibrillation and matched control subjects: incidence and clinical significance. J Am Coll Cardiol. 2008 Oct;52(15):1231-8.

3 Haïssaguerre M, Derval N, Sacher F, Jesel L, Deisenhofer I, de Roy L, et al. Sudden cardiac arrest associated with early repolarization. $\mathrm{N}$ Engl J Med. 2008 May;358(19):2016-23.

4 Tikkanen JT, Anttonen O, Junttila MJ, Aro AL, Kerola T, Rissanen HA, et al. Long-term outcome associated with early repolarization on electrocardiography. N Engl J Med. 2009 Dec;361(26):2529-37.

5 Tikkanen JT, Junttila MJ, Anttonen O, Aro AL, Luttinen S, Kerola T, et al. Early repolarization: electrocardiographic phenotypes associated with favorable long-term outcome. Circulation. 2011 Jun;123(23):2666-73.

6 Xu S, Yang L, Hong D, Chen L, Wang X. The prognostic value of early repolarization pattern for the ventricular tachyarrhythmias of acute myocardial infarction patients: A meta-analysis. Cardiology. DOI: 10.1159/000501474.

7 Perez MV, Friday K, Froelicher V. Semantic confusion: the case of early repolarization and the J point. Am J Med. 2012 Sep;125(9):843-4.

8 Macfarlane PW, Antzelevitch C, Haissaguerre M, Huikuri HV, Potse M, Rosso R, et al. The Early Repolarization Pattern: A Consensus Paper. J Am Coll Cardiol. 2015 Jul; 66(4):470-7.

9 Antzelevitch C, Yan GX. J wave syndromes. Heart Rhythm. 2010 April;7(4):549-558.

10 Stevenson WG, Khan H, Sager P, Saxon LA, Middlekauff HR, Natterson PD, et al. Identification of reentry circuit sites during catheter mapping and radiofrequency ablation of ventricular tachycardia late after myocardial infarction. Circulation. 1993 Oct;88(4 Pt 1): 1647-70.

11 Aizawa Y, Jastrzebski M, Ozawa T, KaweckaJaszcz K, Kukla P, Mitsuma W, et al. Characteristics of electrocardiographic repolarization in acute myocardial infarction complicated by ventricular fibrillation. J Electrocardiol. 2012 May-Jun;45(3):252-9.

12 Long M, Yang L, Huang G, Liu L, Dong Y, Du $Z$, et al. Thrombin and its receptor enhance ST-segment elevation in acute myocardial in- farction by activating the KATP channel. Mol Med. 2010 Jul-Aug;16(7-8):322-32.

13 Naruse Y, Tada H, Harimura Y, Ishibashi M, Noguchi Y, Sato A, et al. Early repolarization increases the occurrence of sustained ventricular tachyarrhythmias and sudden death in the chronic phase of an acute myocardial infarction. Circ Arrhythm Electrophysiol. 2014 Aug;7(4):626-32.

14 Park YM, Cha MS, Kim SH, Kim SJ, Lee SI, Jeon YB, Park CH, Kim YH, Choi IS: Early repolarization is associated with atrial and ventricular tachyarrhythmias in patients with acute St elevation

15 Chen Q, Zheng M, Liu G, Shi X, Zhang R, Zhou X, Xi Y, Sun J, Zhu C, Chen Y, Cheng J, Yin J: Early Repolarization Pattern Predicts the Increased Risk of Ventricular Arrhythmias in Patients With Acute Anterior ST-Segment Elevation Myocardial Infarction - A Propensity Analysis. Circ J. 2017;81(9):1346-1353.

16 Ozcan KS, Güngör B, Tatlısu MA, Osmonov D, Ekmekçi A, Çalık AN, et al. Presence of early repolarization on admission electrocardiography is associated with long-term mortality and MACE in patients with STEMI undergoing primary percutaneous intervention. J Cardiol. 2014 Sep;64(3):164-70. 